\title{
LACK OF SYMMETRY IN LINEAR DETERMINACY DUE TO CONVECTIVE EFFECTS IN REACTION-DIFFUSION-CONVECTION PROBLEMS
}

\author{
A. AL-KIFFAI AND E. C. M. CROOKS
}

\begin{abstract}
This paper is concerned with linear determinacy in monostable reactiondiffusion-convection equations and co-operative systems. We present sufficient conditions for minimal travelling-wave speeds (equivalent to spreading speeds) to equal values obtained from linearisations of the travelling-wave problem about the unstable equilibrium. These conditions involve both reaction and convection terms. We present separate conditions for non-increasing and non-decreasing travelling waves, called 'right' and 'left' conditions respectively, because of the asymmetry in propagation caused by the convection terms. We also give a necessary condition on the reaction term for the existence of convection terms such that both the right and left conditions can be satisfied simultaneously. Examples show that our sufficient conditions for linear determinacy are not necessary and compare these conditions in the scalar case with alternative conditions observed in Malaguti-Marcelli [15] and Benguria-Depassier-Mendez [3]. We also illustrate, for both an equation and a system, the existence of reaction and (non-trivial) convection terms for which the right and left linear determinacy conditions are simultaneously satisfied. An example is given of an equation which is right but not left linearly determinate.
\end{abstract}

\section{Introduction}

The reaction-diffusion equation $u_{t}=d u_{x x}+f(u)$ is well-known as a simple model of phenomena in, for instance, population growth, chemical reaction, flame propagation, etc. For the classical Fisher case [6], $f(u)=r u(1-u)$, Kolmogorov, Petrowskii and Piscounov [10] showed that there exist non-increasing travelling fronts, joining the equilibria 1 and 0 , for all speeds $c \geq 2 \sqrt{d r}$. In many applications, however, there is convective motion in addition to diffusion and reaction, which can have a major impact on the behaviour of solutions. An example of such convection terms arises in a simple one-dimensional model of the motion

Received April 02, 2015, accepted November 11, 2015. 2010 Mathematics Subject Classification. Primary 421305, 42B08.

Key words and phrases. reaction-diffusion-convection systems, travelling waves, spreading speed, linear determinacy, co-operative systems, role of convection.

Corresponding author: E. C. M. Crooks. 
of chemotactic cells, based on a model of Keller and Segel [9]. This model is presented in Benguria, Depassier and Mendez [2], where $\rho$ denotes the density of bacteria chemotactic to a single chemical element of concentration $s$, the density evolves according to $\rho_{t}=\left[D \rho_{x}-\right.$ $\left.\rho \chi s_{x}\right]_{x}+f(\rho), D$ is a diffusion constant and $\chi$ is the chemotactic sensitivity. For travelling front solutions, $s=s(x-c t), \rho=\rho(x-c t)$, we have $s_{t}=-c s_{x}, s_{x}=K \rho / c$, and the problem then reduces to a single differential equation for $\rho$, namely

$$
\rho_{t}=D \rho_{x x}-\frac{\chi K}{c}(\rho)_{x}^{2}+f(\rho) .
$$

Motivated by such models, we first consider a reaction-diffusion-convection equation of form

$$
u_{t}+h^{\prime}(u) u_{x}=u_{x x}+f(u)
$$

with a monostable reaction term $f(u)$ in which 0 is an unstable equilibrium, there is a stable equilibrium $\beta>0$, and there are no equilibria of $f$ between 0 and $\beta$. When $h^{\prime}(u) \equiv 0$, Hadeler and Rothe [8] showed that there exist non-increasing travelling fronts $u(x, t)=w(x-c t)$ with $w(-\infty)=\beta, w(+\infty)=0,0 \leq w \leq \beta$ for (1.1) of all speeds $c \geq c_{0}$, and gave a variational formula for the minimal travelling wave speed $c_{0}$. Here we begin by presenting a straightforward generalisation of this formula to the reaction-diffusion-convection equation (1.1). This minimal speed $c_{0}$ is bounded below by a critical parameter $\bar{c} \in \mathbb{R}$, which we refer to as the 'linear value'. This linear value is obtained from the linearisation of the travelling-wave equation for (1.1) about the unstable equilibria 0 . We present a sufficient condition to guarantee that the minimal wave speed $c_{0}$ equals the linear value $\bar{c}=h^{\prime}(0)+2 \sqrt{f^{\prime}(0)}$, extending [8, Corollary 9] to now involve both the functions $f$ and $h$. This condition is

$$
h^{\prime}(u)+\frac{f(u)}{u \sqrt{f^{\prime}(0)}} \leq h^{\prime}(0)+\sqrt{f^{\prime}(0)} \quad \text { for all } u \in(0,1),
$$

which generalises the classical Hadeler-Rothe condition,

$$
f(u) \leq u f^{\prime}(0) \quad \text { for all } u \in(0,1),
$$

that applies when $h^{\prime}(u) \equiv 0$. Example 2.4 shows that (1.2) is sufficient but not necessary for linear determinacy. Benguria, Depassier and Mendez [3] give an alternative condition to ensure that $c_{0}=\bar{c}$ which again involves both functions $f$ and $h$ and is based on an alternative variational expression from which the minimal travelling wave speed can be estimated. We give examples to show that for a given reaction function $f$, it is possible for the condition in [3] to be satisfied when our condition (1.2) is not satisfied, and vice versa, that (1.2) can be satisfied when the condition in [3] is not. Note here that Gilding and Kersner [7] also discuss extension of the ideas of Hadeler and Rothe [8] to (1.1) in their detailed and substantial 
discussion of an integral equation framework for travelling fronts. Moreover, Malaguti and Marcelli [15] also observe a condition which, in particular, guarantees that $c_{0}=\bar{c}$ if the function $f$ satisfies (1.3) and $\sup _{0<u<1} h^{\prime}(u)=h^{\prime}(0)$ (see also the discussion following Proposition 2.1 in Section 2 below). Example 2.6 illustrates that our condition (1.2) can be satisfied by reaction and convection terms $f$ and $h$ when the function $f$ does not satisfy the classical Hadeler-Rothe condition (1.3). We also show that for this function $f$, our condition (1.2) can be satisfied when the conditions of Benguria-Depassier-Mendez and Malaguti-Marcelli are not. We mention also that Weinberger [20] recently followed and extended the approach of Hadeler and Rothe [8] to introduce a new condition in the case $h^{\prime}(u) \equiv 0$ that involves replacing $u$ in the right hand side of (1.3) by a suitable choice of function $K(u)$, and briefly discussed such generalised conditions in the presence of $h^{\prime}(u)$, but we do not pursue this approach further here.

Note that for (1.1) with $h^{\prime}(u) \equiv 0$, there is, of course, reflection symmetry, and hence corresponding to a non-increasing travelling-front solution $w(x-c t)$, there is a non-decreasing travelling front $\hat{w}(x+c t)$ with $\hat{w}(+\infty)=\beta, \hat{w}(-\infty)=0,0 \leq \hat{w} \leq \beta$ and $w(\xi)=\hat{w}(-\xi)$. On the other hand, it is clear that the presence of the term $h^{\prime}(u) u_{x}$ will affect propagation speeds and, in particular, will break this symmetry between non-increasing and non-decreasing fronts that exists when $h^{\prime}(u) \equiv 0$.

For (1.1), if we have $c_{0}=\bar{c}$, then we say that the problem is right linearly determinate. In the following, we will write $c_{r}^{0}, \bar{c}_{r}$ in place of $c_{0}, \bar{c}$, so that (1.1) is right linearly determinate if $c_{r}^{0}=\bar{c}_{r}$. Correspondingly, we say that (1.1) is left linearly determinate when the critical travelling wave speed for non-decreasing travelling fronts $c_{l}^{0}$ equals the speed $\bar{c}_{l}$ obtained from the linearisation of the travelling wave equation (1.1) about the unstable state, this time with the leading edge tending to the equilibrium at $-\infty$ instead of $+\infty$, in which case $\bar{c}_{l}=h^{\prime}(0)-2 \sqrt{f^{\prime}(0)}$. In general, for given functions $f$ and $h$, it is of course possible that (1.2) is satisfied but the corresponding condition for left linear determinacy is not, and indeed, a problem may be right but not left linearly determinate, or vice versa - see Examples 2.7 and 3.15 below. Linear determinacy for propagation into an unstable state means that the spread rate in the full nonlinear model equals the spread rate in the corresponding travelling-wave problem linearised about the unstable state, which is the speed associated with the leading edge of the wave. It is useful to determine conditions that ensure (right and/or left) linear determinacy because it is easier to calculate a minimal wave speed if it equals the corresponding linear value. Moreover, the minimal wave speed, being equal to a spreading speed, is important for applications to, for example, predicting the speed of spread of biological invasions. For further background and results on linear determinacy, that focusses mainly on problems without convection, see, for instance, [8], [21], and also [4], [12], [13]. 
We then turn to study a co-operative system of two equations of the form

$$
u_{i, t}+h_{i}^{\prime}\left(u_{i}\right) u_{i, x}=d_{i} u_{i, x x}+f_{i}(u), \quad i=1,2 \text {, }
$$

with a reaction term $f(u)$ that satisfies $\frac{\partial f_{i}}{\partial u_{j}} \geq 0$ for all $i \neq j$. For precise assumptions on the equilibria $f$, see hypotheses $s_{2}$ and $s_{6}$ in section 3 . We consider a non-increasing travelling wave solution $w(x, t)=w(x-c t)$ that connects equilibria of $f$ and converges to a co-existence equilibrium $\beta$, with both components positive, at $-\infty$, and to either the equilibrium 0 , or an equilibrium other than 0 , at $+\infty$. Note that the diagonal form $h_{i}^{\prime}\left(u_{i}\right) u_{i, x}$ of our convection term, together with the co-operative assumption $\frac{\partial f_{i}}{\partial u_{j}} \geq 0, i \neq j$, ensures that a comparison principle holds for the system (1.4). We focus here mainly on reaction terms $f$ for which $f^{\prime}(0)$ has the reducible form $\left(\begin{array}{ll}\alpha & 0 \\ \varrho & \sigma\end{array}\right)$ where $\alpha, \varrho>0$ and $\alpha>\sigma$, motivated by models of interaction of distinct species (see [21]). We extend the above theory for the scalar equation (1.1) on the existence of a minimum travelling wave speed $c_{r}^{0}$ to such a system (1.4) by modifying a result of Li, Weinberger and Lewis [11]. This value $c_{r}^{0}$ can be characterised as a slowest spreading speed, in the sense of slowest spreading speed defined in [11, (2.4)]. Hence arguments which extend those in Weinberger, Lewis and Li [21, Theorem 4.2] then yield a sufficient condition that ensures that the minimal speed $c_{r}^{0}$ equals the linear value $\bar{c}$ in this case. Our condition, which generalises (1.2) in the scalar case, is that for all positive $\rho$ and $i=1,2$,

$$
f_{i}\left(\rho \zeta^{r}\left(\bar{\mu}_{r}\right)\right) \leq \rho \bar{\mu}_{r}\left[h_{i}^{\prime}(0)-h_{i}^{\prime}\left(\rho \zeta_{i}^{r}\left(\bar{\mu}_{r}\right)\right)\right] \zeta_{i}^{r}\left(\bar{\mu}_{r}\right)+\rho\left(f^{\prime}(0) \zeta^{r}\left(\bar{\mu}_{r}\right)\right)_{i},
$$

where $\zeta^{r}\left(\bar{\mu}_{r}\right)$ is a certain strictly positive eigenvector of the coefficient matrix $C_{r}(\mu)$, defined in (3.3) below, and $\bar{\mu}_{r}$ is the value of $\mu>0$ at which the infimum in definition (3.6) is attained. This coefficient matrix $C_{r}(\mu)$ is obtained from the linearisation of the travelling-wave problem for the system (1.4) about the unstable equilibria 0 at $+\infty$. We refer to this condition as the right combined condition since it involves a combination of the functions $f$ and $h$. Clearly (1.5) extends [21, (4.9)] and reduces to it when $h^{\prime}(u) \equiv 0$. There is a corresponding condition for non-decreasing travelling-front solutions of (1.4), called the left combined condition, that ensures the system (1.4) is left linearly determinate, namely that for all positive $\rho$ and $i=1,2$,

$$
f_{i}\left(\rho \zeta^{l}\left(\bar{\mu}_{l}\right)\right) \leq \rho \bar{\mu}_{l}\left[h_{i}^{\prime}\left(\rho \zeta_{i}^{l}\left(\bar{\mu}_{l}\right)\right)-h_{i}^{\prime}(0)\right] \zeta_{i}^{l}\left(\bar{\mu}_{l}\right)+\rho\left(f^{\prime}(0) \zeta^{l}\left(\bar{\mu}_{l}\right)\right)_{i},
$$

where $\zeta^{l}\left(\bar{\mu}_{l}\right)$ is a certain strictly positive eigenvector of the coefficient matrix $C_{l}(\mu)$, defined in (3.5) below, which is obtained from the linearisation of the travelling wave problem for (1.4) about 0 at $-\infty$, and $\bar{\mu}_{l}$ is the value of $\mu>0$ at which the infimum in definition (3.7) is attained, see Theorems 3.8, 3.9. Finally, we present a sufficient and necessary condition on $f$ for the existence of a continuously differentiable function $h$ such that (1.5) and (1.6) can be satisfied. 
We conclude by presenting examples to illustrate that for certain given functions $f: \mathbb{R} \rightarrow \mathbb{R}$ and $f: \mathbb{R}^{2} \rightarrow \mathbb{R}^{2}$, we can find functions $h$ so that both the right and left combined conditions (1.5) and (1.6) are satisfied.

\section{A formula for the minimal travelling wave speed of a reaction-diffusion-convection equation}

Consider a reaction-diffusion-convection equation

$$
u_{t}+h^{\prime}(u) u_{x}=u_{x x}+f(u), \quad x \in \mathbb{R}, \quad t \in(0, \infty),
$$

where $u: \mathbb{R} \times[0, \infty) \rightarrow \mathbb{R}$ and the functions $f$ and $h$ satisfy the hypotheses

$e_{1}: \quad f \in C^{1}[0,1]$ and $h \in C^{2}[0,1]$;

$e_{2}: \quad f(0)=f(1)=0, \quad f(u)>0$ for $u \in(0,1)$;

$e_{3}: f^{\prime}(0)>0, \quad f^{\prime}(1)<0$.

A travelling front is a solution $u$ of (2.1) such that $u(x, t)=w(x-c t)$, where $w$ is here taken to be a non-increasing function such that

$$
w(-\infty)=1, \quad w(\infty)=0, \quad 0 \leq w \leq 1
$$

and the speed $c \in \mathbb{R}$ is a constant. Clearly $w$ and $c$ satisfy the ordinary differential equation

$$
-w^{\prime \prime}=c w^{\prime}-h^{\prime}(w) w^{\prime}+f(w)
$$

It is shown in Hadeler and Rothe [8] that when $h^{\prime} \equiv 0$ a travelling front satisfying (2.2) exists if and only if $c \geq c_{r}^{0}$, with

$$
c_{r}^{0}=\inf _{\psi \in \Lambda} \sup _{0<w<1}\left\{\psi^{\prime}(w)+\frac{f(w)}{\psi(w)}\right\}
$$

where the set $\Lambda$ is defined by

$$
\begin{array}{r}
\Lambda:=\left\{\psi:[0,1] \rightarrow[0, \infty): \psi \text { is continuously differentiable, } \psi(0)=0, \psi^{\prime}(0)>0\right. \\
\text { and } \psi(w)>0 \text { for } w \in(0,1)\} .
\end{array}
$$

The following proposition generalises this result to the equation (2.1). Since the proof is similar to that in [8, Theorem 5], we omit it here. This extension of [8] is also observed by Gilding and Kersner [7, Theorem 8.2], and additionally follows from the special case of [5, Lemmas $2.1,2.2]$ when there is only one equation. 
Proposition 2.1. There exists a decreasing travelling-front solution of (2.1) that satisfies properties (2.2) for all speeds $c \geq c_{r}^{0}$, where $c_{r}^{0}$ is characterised by

$$
c_{r}^{0}=\inf _{\psi \in \Lambda} \sup _{0<w<1}\left\{\psi^{\prime}(w)+h^{\prime}(w)+\frac{f(w)}{\psi(w)}\right\},
$$

and $\Lambda$ is as defined in (2.3).

The variational formula (2.4) clearly yields upper bounds for $c_{r}^{0}$ using specific choices of test functions $\psi \in \Lambda$. In particular, if we define

$$
L=\sup _{0<w<1}\left\{\frac{f(w)}{w}\right\}, \quad J=\sup _{0<w<1}\left\{h^{\prime}(w)\right\},
$$

then as in the proof of [8, Corollary 9], and noted also by Malaguti and Marcelli [15], minimising the expression $\sup _{0<w<1}\left\{\psi_{k}^{\prime}(w)+h^{\prime}(w)+\frac{f(w)}{\psi_{k}(w)}\right\}$ with respect to $k>0$, where $\psi_{k} \in \Lambda$ is defined by $\psi_{k}(w)=k w$, gives that

$$
c_{r}^{0} \leq 2 \sqrt{L}+J
$$

Moreover, the value of the minimal speed $c_{r}^{0}$ is bounded below by a critical parameter $\bar{c}_{r} \in \mathbb{R}$ known as the "linear value", which can be defined by the property that the travelling-wave problem linearised about the unstable equilibrium 0 has a real negative eigenvalue if and only if $c \geq \bar{c}_{r}$. Such an eigenvalue $\lambda$ satisfies the equation $\lambda^{2}+\lambda\left(c-h^{\prime}(0)\right)+f^{\prime}(0)=0$, so that such $\lambda$ exists if $c-h^{\prime}(0) \geq 2 \sqrt{f^{\prime}(0)}$. Since $\bar{c}_{r}$ is the smallest speed for which such an eigenvalue exists, we have

$$
\bar{c}_{r}=2 \sqrt{f^{\prime}(0)}+h^{\prime}(0) \leq c_{r}^{0}
$$

and thus

$$
\bar{c}_{r} \leq c_{r}^{0} \leq 2 \sqrt{L}+J .
$$

This estimate clearly yields a set of sufficient conditions, generalising [8, Corollary 9] to the case of (2.1) with $h \neq 0$, that guarantee that the linear value $\bar{c}_{r}$ equals the minimal wave speed $c_{r}^{0}$, namely if

$$
2 \sqrt{L}+J \leq 2 \sqrt{f^{\prime}(0)}+h^{\prime}(0),
$$

then $c_{r}^{0}=\bar{c}_{r}$. In particular, $\bar{c}_{r}=c_{r}^{0}$ if

$$
\sup _{0<w<1} \frac{f(w)}{w}=f^{\prime}(0) \text { and } \sup _{0<w<1} h^{\prime}(w)=h^{\prime}(0) .
$$

The following proposition gives an alternative sufficient condition (2.9) that again ensures $\bar{c}_{r}=c_{r}^{0}$. Note that if (2.8) holds then (2.9) is satisfied, whereas we will show in Example 2.6 that (2.9) can hold when even (2.7) does not hold. 
Proposition 2.2. A sufficient condition to guarantee that the linear value $\bar{c}_{r}=2 \sqrt{f^{\prime}(0)}+h^{\prime}(0)$ for problem (2.1) is equal to the minimal speed $c_{r}^{0}$ is that

$$
h^{\prime}(u)+\frac{f(u)}{\sqrt{f^{\prime}(0)} u} \leq h^{\prime}(0)+\sqrt{f^{\prime}(0)} \quad \text { for all } u \in(0,1) .
$$

Proof. Define a continuous function $y:[0,1] \rightarrow \mathbb{R}$ by

$$
y(u)=\left\{\begin{array}{cc}
\sqrt{f^{\prime}(0)}+h^{\prime}(u)+\frac{f(u)}{\sqrt{f^{\prime}(0)} u} \text { for all } u \in(0,1] \\
2 \sqrt{f^{\prime}(0)}+h^{\prime}(0) & \text { if } \quad u=0 .
\end{array}\right.
$$

Then Proposition 2.1, using the function $\psi(w):=\sqrt{f^{\prime}(0)} w$ in (2.4), and (2.9) together imply that

$$
c_{r}^{0} \leq \sup _{0<w<1}\{y(w)\} \leq y(0)
$$

and hence $c_{r}^{0} \leq 2 \sqrt{f^{\prime}(0)}+h^{\prime}(0)=\bar{c}_{r}$, so that $c_{r}^{0}=\bar{c}_{r}$, by (2.6).

Note that for each $k>0$, we can consider the continuous function $y_{k}(u):[0,1] \rightarrow \mathbb{R}$,

$$
y_{k}(u)=\left\{\begin{array}{l}
k+h^{\prime}(u)+\frac{f(u)}{k u} \text { for all } u \in(0,1], \\
k+h^{\prime}(0)+\frac{f^{\prime}(0)}{k} \quad \text { if } \quad u=0 .
\end{array}\right.
$$

Imposing a condition $h^{\prime}(u)+\frac{f(u)}{k u} \leq h^{\prime}(0)+\frac{f^{\prime}(0)}{k}, u \in(0,1)$ clearly yields an analogue of (2.10) with $y$ replaced by $y_{k}$. Therefore, in some sense, the best estimate of this type will be obtained by minimising $k+h^{\prime}(0)+\frac{f^{\prime}(0)}{k}$ with respect to $k$, and this minimum is attained at $k=\sqrt{f^{\prime}(0)}$. In fact, when $k=\sqrt{f^{\prime}(0)}$, the value $y_{k}(0)$ is exactly the linear value, whereas if $k \neq \sqrt{f^{\prime}(0)}$, the estimate $c_{r}^{0} \leq y_{k}(0)$ does not imply linear determinacy.

In particular, (2.9) holds if the condition (2.11) in the following lemma is satisfied, since this ensures that the function $u \mapsto h^{\prime}(u)+\frac{f(u)}{\sqrt{f^{\prime}(0)} u}$ is non-increasing on $(0,1)$.

Lemma 2.3. A sufficient condition to guarantee that the linear value $\bar{c}_{r}=2 \sqrt{f^{\prime}(0)}+h^{\prime}(0)$ equals the minimal speed $c_{r}^{0}$ is

$$
h^{\prime \prime}(u)+\frac{1}{\sqrt{f^{\prime}(0)}}\left\{\frac{f^{\prime}(u)}{u}-\frac{f(u)}{u^{2}}\right\} \leq 0, \quad \text { for all } u \in(0,1) .
$$


The following example, which is a generalisation of [8, Theorem 11] to include convective effects, illustrates that there exist functions $f$ and $h$ for which the sufficient condition (2.9) is not satisfied, but the minimal travelling speed is nevertheless linearly determinate. So (2.9) is sufficient, but not necessary, for linear determinacy.

Example 2.4. Consider the equation

$$
u_{t}=u_{x x}-\beta u u_{x}+\gamma u(1-u)(1+2 \gamma u)
$$

where $\beta=2(\sqrt{\gamma}-\gamma)$ and $0<\gamma<1$. Then $h^{\prime}(u)=\beta u$ and $f(u)=\gamma u(1-u)(1+2 \gamma u)$, so $e_{1}-e_{3}$ are clearly satisfied. Then $\bar{c}_{r}=2 \sqrt{\gamma}$, and condition (2.9) says that for all $u \in(0,1)$,

$$
h^{\prime}(u)+\frac{f(u)}{\sqrt{\gamma} u} \leq \sqrt{\gamma} \Leftrightarrow(1-\sqrt{\gamma}) u+\gamma u(1-u) \leq 0,
$$

which fails to hold even for some $u \in(0,1)$, since $\gamma \in(0,1)$. But $u(x, t):=w(x-c t)$ with $w(\xi):=$ $(1+\exp (\sqrt{\gamma} \xi))^{-1}$ and $c=2 \sqrt{\gamma}$ is easily checked to be an explicit travelling-wave solution of (2.12) that satisfies (2.2). So

$$
2 \sqrt{\gamma}=\bar{c}_{r} \leq c_{r}^{0} \leq 2 \sqrt{\gamma}
$$

and hence $\bar{c}_{r}=c_{r}^{0}$.

An alternative condition that ensures $c_{r}^{0}=\bar{c}_{r}$ is given by Benguria, Depassier and Mendez in [3], namely

$$
\frac{f^{\prime \prime}(u)}{\sqrt{f^{\prime}(0)}}+h^{\prime \prime}(u)<0, \quad \text { for all } u \in(0,1) .
$$

This condition is derived using a different variational characterisation of $c_{r}^{0}$, for which we refer to [3] for details. Note that our convection term $h^{\prime}(u)$ is replaced in [3] by $\mu \phi(u)$, where $\phi$ is a $C^{1}$-function such that, for simplicity, it is assumed that $\phi(0)=0$, but this restriction on $\phi(0)$ clearly only affects the numerical value of $\bar{c}_{r}$, not the condition (2.14), and can be removed. Note that, in fact, another proof of Proposition 2.2 can be obtained using the variational characterisation in [3]; see the discussion below inequality (14) in [3], taking $\alpha=1 / \sqrt{f^{\prime}(0)}$, $h^{\prime}(u)=\mu \phi(u)$ and $h^{\prime}(0)=0$.

The following examples compare our condition (2.9) with (2.14), (2.7) and (2.8), and in particular, illustrate that functions $f$ and $h$ can be found satisfying (2.14) but not (2.9), and, vice versa, that there exist functions which satisfy (2.9) but not (2.14).

Example 2.5. Choose $f(u)=u(1-u)$ and $h(u)=\left(\frac{\delta}{2}\right) u^{2}, \delta \in \mathbb{R}$. Then $f$ satisfies the properties $e_{1}-e_{3}$, and for this function $f$, condition (2.11) says that

$$
h^{\prime \prime}(u) \leq 1 \quad \text { for all } u \in(0,1),
$$


which holds if and only if $\delta \leq 1$. On the other hand, condition (2.14) is satisfied whenever $\delta=h^{\prime \prime}(u)<2$ for all $u \in(0,1)$. Hence if $\delta \in(1,2)$, then (2.14) is satisfied but (2.11) is not, and moreover, it is easy to check that our weaker condition (2.9) is also not satisfied for such $\delta$.

The next example shows that $f$ and $h$ can be found which satisfy condition (2.9) but not (2.14).

Example 2.6. Choose $f(u)=u(1-u)(\delta+u)$, where $\delta>0$. Then $f$ satisfies the properties $e_{1}-e_{3}$, and for this function $f$, condition (2.11) says that

$$
h^{\prime \prime}(u) \leq-\frac{1}{\sqrt{\delta}}\left\{\frac{\delta+2 u-2 \delta u-3 u^{2}}{u}-\frac{u(1-u)(\delta+u)}{u^{2}}\right\}=\frac{1}{\sqrt{\delta}}\{\delta-1+2 u\} \quad \text { for all } u \in(0,1),
$$

whereas condition (2.14) will be satisfied if

$$
h^{\prime \prime}(u)<-\frac{f^{\prime \prime}(u)}{\sqrt{f^{\prime}(0)}}=\frac{1}{\sqrt{\delta}}\{2 \delta-2+6 u\} \quad \text { for all } u \in(0,1) .
$$

Thus for condition (2.11) to be satisfied but (2.14) is not, we need that for some $u \in(0,1)$,

$$
2 \delta-2+6 u<\delta-1+2 u \Leftrightarrow \frac{1-\delta}{4}>u .
$$

So in particular, if we choose $\delta=\frac{1}{2}$, then (2.15) holds for $u \in\left(0, \frac{1}{8}\right)$, and (2.11) holds if

$$
h^{\prime \prime}(u) \leq \frac{1}{\sqrt{\delta}}\{\delta-1+2 u\}=\sqrt{2}\left(2 u-\frac{1}{2}\right) \quad \text { for all } u \in(0,1),
$$

whereas (2.14) does not hold if

$$
\sqrt{2}(6 u-1)=\frac{1}{\sqrt{\delta}}\{2 \delta-2+6 u\}<h^{\prime \prime}(u) \quad \text { for some } u \in(0,1) .
$$

For instance, (2.16) and (2.17) are clearly both satisfied if $h^{\prime \prime}(u) \equiv \sqrt{2}\left(2 u-\frac{1}{2}\right)$, and thus

$$
h(u)=\frac{\sqrt{2}}{3} u^{3}-\frac{1}{2 \sqrt{2}} u^{2}+A u+B, \quad A, B \in \mathbb{R} .
$$

Hence for these functions $f$ and $h$, our condition (2.9) holds but condition (2.14) does not. Note that the Malaguti-Marcelli alternative condition (2.7) also does not hold for this choice of $f$ and $h$, and whenever $\delta<1$, the function $f$ does not satisfy the classical condition (1.3).

Our final example in this section illustrates that, for certain reaction and convection terms $f$ and $h$, the equation (1.1) is right linearly determinate but not left linearly-determinate; that is, $c_{r}^{0}=\bar{c}_{r}$, but $c_{l}^{0} \neq \bar{c}_{l}$. This phenomenon can clearly only occur in the presence of convection, since if $h^{\prime}(u) \equiv 0$, symmetry implies that right and left linear determinacy are equivalent. 
Example 2.7. When $a>2$, the convection-less equation

$$
u_{t}=u_{x x}+u(1-u)(1+a u)
$$

is not linearly determinate [8, Theorem 11]. However, if $f(u)=u(1-u)(1+a u)$, condition (2.9) holds if the convection term is $h^{\prime}(u)=u(1-a)$. Thus

$$
u_{t}=u_{x x}-h^{\prime}(u) u_{x}+u(1-u)(1+a u)
$$

is right linearly determinate. Now note that for a non-decreasing non-negative initial condition $u_{0}$ of (2.18), the solution $u$ of (2.18) is non-negative and satisfies $u_{x} \geq 0$ (see, for instance, [1, Lemma 5.1]). Then since $h^{\prime}(u) \leq 0$ for $u \geq 0$, and $u_{x} \geq 0$, we have

$$
u_{t}=u_{x x}+u(1-u)(1+a u) \leq u_{x x}-h^{\prime}(u) u_{x}+u(1-u)(1+a u),
$$

so $u$ is a sub-solution of (2.19). Thus by Comparison Principle 3.1, the solution $u^{*}$ of (2.19) with a non-decreasing non-negative initial condition $u_{0}$ is greater than or equal to the solution $u$ of (2.18) with the same initial condition $u_{0}$. Therefore the left spreading speed $c_{l}^{*}$ of (2.19) will be less than or equal the left spreading speed $c_{l}$ of (2.18), which is strictly less that $\bar{c}_{l}=-2 \sqrt{f^{\prime}(0)}=-2$ because (2.18) is not linearly determinate. That is,

$$
c_{l}^{*} \leq c_{l}<-2 \sqrt{f^{\prime}(0)}
$$

But since $h^{\prime}(0)=0$, we have $c_{l}<h^{\prime}(0)-2 \sqrt{f^{\prime}(0)}=-2 \sqrt{f^{\prime}(0)}$, and hence (2.19) is not left linearly determinate because

$$
c_{l}^{*}<c_{l}<h^{\prime}(0)-2 \sqrt{f^{\prime}(0)} \text {. }
$$

\section{Sufficient conditions for linear determinacy for reaction-diffusion-convection systems}

Consider a system of reaction-diffusion-convection equations of the form

$$
\begin{aligned}
& u_{1, t}+h_{1}^{\prime}\left(u_{1}\right) u_{1, x}=u_{1, x x}+f_{1}\left(u_{1}, u_{2}\right), \\
& u_{2, t}+h_{2}^{\prime}\left(u_{2}\right) u_{2, x}=d_{2} u_{2, x x}+f_{2}\left(u_{1}, u_{2}\right),
\end{aligned}
$$

where $d_{2}>0$, the reaction term $f=\left(f_{1}\left(u_{1}, u_{2}\right), f_{2}\left(u_{1}, u_{2}\right)\right)$ satisfies the co-operative assumption $\frac{\partial f_{i}}{\partial u_{j}}(u) \geq 0, i \neq j$, the convection function $h$ has the 'diagonal' form $h=\left(h_{1}\left(u_{1}\right), h_{2}\left(u_{2}\right)\right)$, and $u=\left(u_{1}, u_{2}\right) \in \mathbb{R}^{2}$. For $T>0$, denote $\Gamma_{T}=\left\{u: \mathbb{R} \times[0, T] \rightarrow \mathbb{R}^{2}: u\right.$ is bounded, continuous, $u_{t}, u_{x}, u_{x x}$ exist and are continuous on $\left.\mathbb{R} \times(0, T]\right\}$, and for $(x, t) \in \mathbb{R} \times(0, T]$ and $u \in \Gamma_{T}$, define

$$
N(u)(x, t):=-u_{t}(x, t)+A u_{x x}(x, t)-h^{\prime}(u) u_{x}(x, t)+f(u)(x, t)
$$


where $f: \mathbb{R}^{2} \rightarrow \mathbb{R}^{2}, A=\operatorname{diag}\left(1, d_{2}\right)$, and $h^{\prime}(u)=\operatorname{diag}\left(h_{1}^{\prime}\left(u_{1}\right), h_{2}^{\prime}\left(u_{2}\right)\right)$.

The following theorem is a useful tool for system (3.1). Note that, of course, a reactiondiffusion-convection system does not, in general, possess a comparison principle, but the diagonal structure of $h^{\prime}(u)$ and the co-operative assumption on $f$ together ensure that such a principle does hold here.

Theorem 3.1 (Comparison principle). Let the function $f$ satisfy $\frac{\partial f_{i}}{\partial u_{j}}(u) \geq 0, i \neq j$, and $\underline{u}, \bar{u} \in$ $\Gamma_{T}$ be such that $\underline{u}, \bar{u}$ are continuous on $\mathbb{R} \times[0, T], \underline{u}_{x}, \bar{u}_{x}$ are bounded and uniformly continuous on $\mathbb{R} \times(0, T], N(\bar{u})(x, t) \leq 0$, and $N(\underline{u})(x, t) \geq 0$ for $(x, t) \in \mathbb{R} \times(0, T)$. Suppose that $\bar{u}(x, 0) \geq \underline{u}(x, 0)$ for all $x \in \mathbb{R}$. Then $\bar{u}(x, t) \geq \underline{u}(x, t)$, for all $(x, t) \in \mathbb{R} \times[0, T]$.

Proof. This follows from an application of [19, Theorem 5.3], as also noted in [5].

Recall that a matrix is said to be reducible if it can be put into lower block triangular form by reordering the coordinates and, if this cannot be done, the matrix is said to be irreducible. If all the diagonal blocks in the lower block triangular form of a reducible matrix are irreducible, this is said to be in Frobenius form. The statement of Perron-Frobenius theorem is the following.

Theorem 3.2 (Perron-Frobenius [17]). Any non-zero irreducible matrix with non-negative entries has a unique positive eigenvalue, called the principal eigenvalue, which has a corresponding strictly positive principal eigenvector. In addition, the absolute values of all the other eigenvalues are less than the principal eigenvalue.

The following is a useful corollary for an irreducible matrix with non-negative off-diagonal entries.

Corollary 3.3. Given any irreducible matrix with off-diagonal entries non-negative, there exists a unique real eigenvalue, called the principal eigenvalue, with a corresponding strictly positive principal eigenvector. In addition, the real parts of all other eigenvalues are strictly less than the principal eigenvalue.

Proof. Let $M$ be an irreducible matrix with non-negative off-diagonal entries. Then there exists $\varepsilon>0$ such that $M+\varepsilon I$ is a non-zero irreducible matrix with non-negative entries. By Theorem 3.2, there exists a positive eigenvalue $\lambda$ with positive eigenvector $q$ such that $(M+$ $\varepsilon I) q=\lambda q$. Then $M q=(\lambda-\varepsilon) q$, so $M$ has a real eigenvalue $\lambda-\varepsilon$ with positive eigenvector $q$. Moreover, if $\mu$ is an eigenvalue of $M+\varepsilon I$ other than $\lambda$, then $|\mu|<\lambda$, so if $v$ is an eigenvalue of the matrix $M$ other than $\lambda-\varepsilon$, then $|v+\varepsilon|<\lambda$, which implies that $\operatorname{Re}(v)<\lambda-\varepsilon$.

For $a, b \in \mathbb{R}^{n}$, we say $a<b$ (respectively $a \leq b$ ) to mean that $a_{k}<b_{k}$ (resp. $a_{k} \leq b_{k}$ ) for each $k \in\{1,2, \ldots, n\}$, and for a vector $v \in \mathbb{R}^{n}$, we say that $v \in[a, b]$ if $a_{k} \leq v_{k} \leq b_{k}$ for all $k$. For 
ease of exposition, we discuss a system of two equations here, and note that similar results hold for a system of $n$ equations, as will be discussed in [1].

We assume in the following that the functions $f: \mathbb{R}^{2} \rightarrow \mathbb{R}^{2}$ and $h: \mathbb{R}^{2} \rightarrow \mathbb{R}^{2}$ in system (3.1) satisfy the hypotheses

$s_{1}: \frac{\partial f_{i}}{\partial u_{j}} \geq 0$ for $i \neq j$;

$s_{2}: f(0)=0$, there exists $\beta>0$ such that $f(\beta)=0$, and there is no $v>0$ other than $\beta$ such that $f(v)=0$ and $0<v \leq \beta$;

$s_{3}$ : Neither $f$ nor $h$ depends explicitly on either $x$ or $t$, and $d_{2}>0$ is constant;

$s_{4}: h=\left(h_{1}\left(u_{1}\right), h_{2}\left(u_{2}\right)\right)$;

$s_{5}$ : The functions $f$ and $h$ are continuously differentiable at $v$ for each $0 \leq v \leq \beta$;

$s_{6}$ : The Jacobian matrix $f^{\prime}(0)$ is in Frobenius form and such that the principal eigenvalue of the upper left diagonal block of $f^{\prime}(0)$ is positive and, if there are two blocks, is strictly larger than the principal eigenvalue of the other diagonal block, and the entry to the left of the second diagonal block is strictly positive.

\section{Remark 3.4.}

(i) Property $s_{1}$ says that the system is co-operative, and is thus order-preserving, by the comparison principle Theorem 3.1.

(ii) Property $s_{6}$ implies that $f^{\prime}(0)$ has one of two forms. In the first case, $f^{\prime}(0)=\left(\begin{array}{ll}\alpha & \delta \\ \varrho & \sigma\end{array}\right)$ where $\delta, \varrho>0$, and then Corollary 3.3 implies that the principal eigenvalue is strictly positive and has a strictly positive principal eigenvector. The second case is when $f^{\prime}(0)=\left(\begin{array}{ll}\alpha & 0 \\ \varrho & \sigma\end{array}\right)$ where $\alpha, \varrho>0$ and $\alpha>\sigma$, in which case it is easy to show that the eigenvector of $f^{\prime}(0)$ corresponding to the principal eigenvalue $\alpha$ of the first block is strictly positive. Indeed, suppose the eigenvector of $f^{\prime}(0)$ corresponding to the principal eigenvalue $\alpha$ is $z=(x, y)^{T}$. Then $\varrho x+\sigma y=\alpha y \Leftrightarrow \varrho x=(\alpha-\sigma) y$. Since $\alpha>\sigma$ and $\varrho>0$ it follows that $x$ and $y$ have the same sign, and thus can be chosen so that $z=(x, y)^{T}$ is strictly positive. Note that in all of our examples below, we focus on this second case, which is motivated by models of interaction of separate species and for which calculations are relatively tractable.

(iii) We present $s_{6}$ in a form that clearly extends to the case when the system (3.1) is replaced by a similar system of $n$ equations that satisfy Hypotheses $s_{1}-s_{5}$, when there might be more than two diagonal blocks in $f^{\prime}(0)$. See, for example, [21], [1]. In this general case, $\left[18\right.$, Theorem 2.1] ensures that the eigenvector of $f^{\prime}(0)$ corresponding to the principal eigenvalue of the first block is strictly positive. 
The linearisation of the system (3.1) at 0 gives

$$
u_{i, t}+h_{i}^{\prime}(0) u_{i, x}=d_{i} u_{i, x x}+\left(f^{\prime}(0) u\right)_{i}, \quad i=1,2 .
$$

Applying the method of separation of variables then shows that the solution of the system (3.2) with initial condition $e^{-\mu x} \alpha$, where $\mu \in \mathbb{R}$ and $\alpha \in \mathbb{R}^{2}$ are constant, has the form $e^{-\mu x} \eta(t)$, where the vector-valued function $\eta$ is a solution of the system $\eta_{t}=C_{r}(\mu) \eta$ with $\eta(0)=\alpha$, where the coefficient matrix $C_{r}(\mu)$ is

$$
C_{r}(\mu)=\mu^{2} \operatorname{diag}\left(1, d_{2}\right)+\mu \operatorname{diag}\left(h_{1}^{\prime}(0), h_{2}^{\prime}(0)\right)+f^{\prime}(0),
$$

which has non-negative off-diagonal entries, by property $s_{1}$.

In order to consider non-decreasing travelling fronts as well as non-increasing travelling fronts, it is useful to remark next that if we define $\hat{u}(x, t)=u(-x, t)$ where $u$ is a solution of system (3.1), then $\hat{u}$ is a solution of the system

$$
\begin{gathered}
u_{1, t}-h_{1}^{\prime}\left(u_{1}\right) u_{1, x}=u_{1, x x}+f_{1}\left(u_{1}, u_{2}\right) \\
u_{2, t}-h_{2}^{\prime}\left(u_{2}\right) u_{2, x}=d_{2} u_{2, x x}+f_{2}\left(u_{1}, u_{2}\right)
\end{gathered}
$$

for which the related coefficient matrix is

$$
C_{l}(\mu)=\mu^{2} \operatorname{diag}\left(1, d_{2}\right)-\mu \operatorname{diag}\left(h_{1}^{\prime}(0), h_{2}^{\prime}(0)\right)+f^{\prime}(0) .
$$

Clearly system (3.4) is obtained from system (3.1) simply by replacing $h$ by $\hat{h}:=-h$, and $f$ and $\hat{h}$ satisfy hypotheses $s_{1}-s_{6}$ if and only if these hypotheses hold for $f$ and $h$. So results for non-decreasing travelling-front solutions of (3.1) can be deduced immediately from results on non-increasing front solutions of (3.4).

Now let $\gamma_{\sigma}^{r}$ denote the principal eigenvalue of the $\sigma$ th block for the matrix $C_{r}(\mu)$. Following [21], we define

$$
\bar{c}_{r}:=\inf _{\mu>0}\left\{\frac{\gamma_{1}^{r}(\mu)}{\mu}\right\} .
$$

Note that we again use the notation $\bar{c}_{r}$ here because in the case when $f^{\prime}(0)$ is actually irreducible (so has only one irreducible block in the Frobenius form), it is easy to see that this definition of $\bar{c}_{r}$ coincides with the natural extension to the system (3.1) of the definition of $\bar{c}_{r}$ in the scalar case discussed in section 2. Note also that the travelling-wave problem linearised about the unstable equilibrium 0 has a real negative eigenvalue corresponding to a strictly positive eigenvector if and only if $c \geq \bar{c}_{r}$, and in fact, (3.6) yields an alternative characterisation of $\bar{c}_{r}$ in the scalar case also. 
The following lemma gives a sufficient condition for $\bar{\mu}_{r}$, defined to be the value of $\mu>0$ at which the infimum in the definition of $\bar{c}_{r}$ is attained, to equal $\bar{\mu}_{l}$, the value at which the infimum in the definition of

$$
\bar{c}_{l}:=-\inf _{\mu>0}\left\{\frac{\gamma_{1}^{l}(\mu)}{\mu}\right\}
$$

is attained, where $\gamma_{\sigma}^{l}$ is the principal eigenvalue of the $\sigma$ th block of $C_{l}(\mu)$. Here and in the following, we denote by $\zeta^{r}\left(\bar{\mu}_{r}\right)$ an eigenvector of $C_{r}(\mu)$ corresponding to the eigenvalue $\gamma_{1}^{r}\left(\bar{\mu}_{r}\right)$ and by $\zeta^{l}\left(\bar{\mu}_{l}\right)$ an eigenvector of $C_{l}(\mu)$ corresponding to the eigenvalue $\gamma_{1}^{l}\left(\bar{\mu}_{l}\right)$.

Lemma 3.5. If $f$ and $h$ satisfy hypotheses $s_{1}-s_{6}$, and $f^{\prime}(0)$ is as in the second case in Remark 3.4 (ii), then (i) $\bar{\mu}_{r}=\bar{\mu}_{l}$, and (ii) the eigenvector $\zeta^{r}\left(\bar{\mu}_{r}\right)$ of $C_{r}(\mu)$ corresponding to $\gamma_{1}^{r}\left(\bar{\mu}_{r}\right)$ can be chosen equal to the eigenvector $\zeta^{l}\left(\bar{\mu}_{l}\right)$ of $C_{l}(\mu)$ corresponding to $\gamma_{1}^{l}\left(\bar{\mu}_{l}\right)$ if and only if $h_{1}^{\prime}(0)=$ $h_{2}^{\prime}(0)$.

Proof. Part (i) is immediate from the definitions of $\bar{c}_{r}$ and $\bar{c}_{l}$. For (ii), let $h^{\prime}(0)=\operatorname{diag}\left(h_{1}^{\prime}(0), h_{2}^{\prime}(0)\right)$ and $\zeta^{r}\left(\bar{\mu}_{r}\right)=\left(\begin{array}{ll}1 & \alpha_{2}\end{array}\right)^{T}$. Then corresponding to the eigenvalue $\gamma_{1}^{r}\left(\bar{\mu}_{r}\right)=\bar{\mu}_{r}^{2}+\bar{\mu}_{r} h_{1}^{\prime}(0)+\alpha$, we have

$$
\alpha_{2}=-\varrho /\left(\sqrt{\alpha}\left(h_{2}^{\prime}(0)-h_{1}^{\prime}(0)\right)+\alpha\left(d_{2}-2\right)+\sigma\right)
$$

whereas for the eigenvector $\zeta^{l}\left(\bar{\mu}_{l}\right)=\left(1 \hat{\alpha}_{2}\right)^{T}$ corresponding to the eigenvalue $\gamma_{1}^{l}\left(\bar{\mu}_{l}\right)$, we have

$$
\hat{\alpha}_{2}=-\varrho /\left(\sqrt{\alpha}\left(h_{1}^{\prime}(0)-h_{2}^{\prime}(0)\right)+\alpha\left(d_{2}-2\right)+\sigma\right) .
$$

It is then clear that $\zeta^{r}\left(\bar{\mu}_{r}\right)=\zeta^{l}\left(\bar{\mu}_{l}\right)$ if and only if $h_{1}^{\prime}(0)=h_{2}^{\prime}(0)$.

In the previous section we have a single equation, and have only two equilibria 0 and $\beta$, whereas in this section, we have a system with two equations and, by hypothesis $s_{2}$, may have equilibria in addition to 0 and $\beta$ if they have at least one component zero. For a system with only two equilibria $0, \beta$ with $\beta>0$ and $f^{\prime}(0)$ an irreducible matrix, Lui [14] gave sufficient conditions for spreading speeds to equal linear values. These results were generalised by [21] to systems where the Frobenius form may have multiple diagonal blocks and there may be more equilibria other than 0 and $\beta$ in $[0, \beta]$ provided any additional equilibrium $v$ has $v_{k}=0$ for at least one $k \in\{1,2, \ldots, n\}$.

The following theorem concerns the existence of a value $c_{r}^{0} \in \mathbb{R}$ which can be characterised as a minimum travelling wave speed of system (3.1) in a certain sense. This result, which generalises Proposition 2.2 about the equation (1.1) to the system (3.1), follows easily by adapting the proof of [11, Theorem 4.2]. 
Theorem 3.6. If the system (3.1) satisfies hypotheses $s_{1}-s_{6}$, then there exists $c_{r}^{0} \in \mathbb{R}$ such that for every $c \geq c_{r}^{0}$ this system has a non-increasing travelling wave solution $w(x-c t)$ of speed $c$ with $w(-\infty)=\beta$ and $w(\infty)$ a zero of $f$ other than $\beta$. If there is a travelling wave solution $w(x-c t)$ with $w(-\infty)=\beta$ such that for at least one component $i, \liminf _{x \rightarrow \infty} w_{i}(x)=0$, then $c \geq c_{r}^{0}$.

In fact, it can be shown, similarly to [11], that $c_{r}^{0}$ is actually the slowest spreading speed for the system (3.1) with non-increasing initial data $u(0, x)=u_{0}(x)$ such that $u_{0}(-\infty)=\beta, u_{0}(+\infty)=$ 0 , in the sense of slowest spreading speed defined in $[11,(2.4)]$. This characterisation of the minimal wave speed $c_{r}^{0}$ as the slowest spreading speed enables proof of the following result using a straightforward modification of the ideas in [21, Theorem 4.2].

Theorem 3.7. Suppose that the functions $f$ and $h$ in system (3.1) satisfy hypotheses $s_{1}-s_{6}$. Assume that $\bar{\mu}_{r}$ is finite,

$$
\gamma_{1}^{r}\left(\bar{\mu}_{r}\right)>\gamma_{2}^{r}\left(\bar{\mu}_{r}\right)
$$

and

$$
f_{i}\left(\rho \zeta^{r}\left(\bar{\mu}_{r}\right)\right) \leq \rho \bar{\mu}_{r}\left[h_{i}^{\prime}(0)-h_{i}^{\prime}\left(\rho \zeta_{i}^{r}\left(\bar{\mu}_{r}\right)\right)\right] \zeta_{i}^{r}\left(\bar{\mu}_{r}\right)+\rho\left(f^{\prime}(0) \zeta^{r}\left(\bar{\mu}_{r}\right)\right)_{i} \quad \text { for all } \rho>0, i=1,2 .
$$

Then $c_{r}^{0}=\bar{c}_{r}$, where $c_{r}^{0}$ is the minimum travelling wave speed in Theorem 3.6 and $\bar{c}_{r}$ is the linear value defined in (3.6).

Note that when $f^{\prime}(0)$ is irreducible, condition (3.8) is simply considered to be satisfied trivially.

For non-decreasing travelling-front solutions of system (3.1), the analogues of Theorems 3.6 and 3.7 are the following.

Theorem 3.8. If the system (3.1) satisfies hypotheses $s_{1}-s_{6}$, then there exists $c_{l}^{0} \in \mathbb{R}$ such that for every $c \leq c_{l}^{0}$ the system (3.1) has a non-decreasing travelling wave solution $w(x-c t)$ of speed $c$ with $w(\infty)=\beta$ and $w(-\infty)$ a zero of $f$ other than $\beta$. If there is a travelling wave solution $w(x-c t)$ with $w(\infty)=\beta$ such that for at least one component $i, \liminf _{x \rightarrow-\infty} w_{i}(x)=0$, then $c \leq c_{l}^{0}$.

Theorem 3.9. Suppose that the functions $f$ and $h$ satisfy hypotheses $s_{1}-s_{6}$. Assume that $\bar{\mu}_{l}$ is finite,

$$
\gamma_{1}^{l}\left(\bar{\mu}_{l}\right)>\gamma_{2}^{l}\left(\bar{\mu}_{l}\right)
$$

and

$$
f_{i}\left(\rho \zeta^{l}\left(\bar{\mu}_{l}\right)\right) \leq \rho \bar{\mu}_{l}\left[h_{i}^{\prime}\left(\rho \zeta_{i}^{l}\left(\bar{\mu}_{l}\right)\right)-h_{i}^{\prime}(0)\right] \zeta_{i}^{l}\left(\bar{\mu}_{l}\right)+\rho\left(f^{\prime}(0) \zeta^{l}\left(\bar{\mu}_{l}\right)\right)_{i} \quad \text { for all } \rho>0, i=1,2 .
$$

Then the maximum travelling wave speed $c_{l}^{0}$ equals the linear value $\bar{c}_{l}$ defined in (3.7). 
The following lemma gives a sufficient condition to guarantee that (3.8) and (3.10) hold for the matrices $C_{r}(\mu)$ and $C_{l}(\mu)$ respectively.

Lemma 3.10. Given matrices $f^{\prime}(0)$ and $h^{\prime}(0)$ as in Lemma 3.5, a sufficient condition to have that (3.8) and (3.10) are satisfied for the matrices $C_{r}(\mu)$ and $C_{l}(\mu)$ respectively is that

$$
\frac{\sigma-\alpha\left(2-d_{2}\right)}{\sqrt{\alpha}}<h_{1}^{\prime}(0)-h_{2}^{\prime}(0)<\frac{\alpha\left(2-d_{2}\right)-\sigma}{\sqrt{\alpha}} \text {. }
$$

Then the eigenvectors $\zeta^{r}\left(\bar{\mu}_{r}\right), \zeta^{l}\left(\bar{\mu}_{l}\right)$ corresponding to $\gamma_{1}^{r}\left(\bar{\mu}_{r}\right), \gamma_{1}^{l}\left(\bar{\mu}_{l}\right)$ are strictly positive.

Proof. With $\bar{\mu}_{r}=\bar{\mu}_{l}=\sqrt{\alpha}$, we have $\gamma_{1}^{r}\left(\bar{\mu}_{r}\right)=2 \alpha+h_{1}^{\prime}(0) \sqrt{\alpha}$ and $\gamma_{2}^{r}\left(\bar{\mu}_{r}\right)=\alpha d_{2}+h_{2}^{\prime}(0) \sqrt{\alpha}+$ $\sigma$. So $\gamma_{1}^{r}\left(\bar{\mu}_{r}\right)>\gamma_{2}^{r}\left(\bar{\mu}_{r}\right)$ if and only if $h_{1}^{\prime}(0)-h_{2}^{\prime}(0)>\left(\sigma-\alpha\left(2-d_{2}\right)\right) / \sqrt{\alpha}$. On the other hand, $\gamma_{1}^{l}\left(\bar{\mu}_{l}\right)=2 \alpha-h_{1}^{\prime}(0) \sqrt{\alpha}$ and $\gamma_{2}^{l}\left(\bar{\mu}_{l}\right)=\alpha\left(d_{2}\right)-h_{2}^{\prime}(0) \sqrt{\alpha}+\sigma$, so that $\gamma_{1}^{l}\left(\bar{\mu}_{l}\right)>\gamma_{2}^{l}\left(\bar{\mu}_{l}\right)$ if and only if $h_{1}^{\prime}(0)-h_{2}^{\prime}(0)<\left(\alpha\left(2-d_{2}\right)-\sigma\right) / \sqrt{\alpha}$. Then straightforward calculation shows that (3.12) ensures the eigenvectors $\zeta^{r}\left(\bar{\mu}_{r}\right), \zeta\left(\bar{\mu}_{l}\right)$ are strictly positive, since $\varrho>0$ and (3.8), (3.10) hold (note the analogous observation for eigenvectors of $f^{\prime}(0)=C_{r}(0)$ already mentioned in Remark 3.4 (ii)).

Remark 3.11. Clearly (3.9) in Theorem 3.7 reduces to the condition (2.9) for the scalar case in Proposition 2.2, because $\bar{\mu}_{r}=\sqrt{f^{\prime}(0)}, \zeta^{r}\left(\bar{\mu}_{r}\right)=1$. So setting $\rho=\rho \zeta^{r}\left(\bar{\mu}_{r}\right)=u$ in (2.9) gives

$$
h^{\prime}(\rho)+\frac{f(\rho)}{\rho \bar{\mu}_{r}} \leq h^{\prime}(0)+\frac{f^{\prime}(0)}{\bar{\mu}_{r}}, \quad \text { for all } \rho \in(0,1),
$$

which is precisely (3.9).

The next proposition gives a necessary condition for existence of a function $h$ satisfying both the 'right' and 'left' combined conditions,

$$
f_{i}\left(\rho \zeta^{r}\left(\bar{\mu}_{r}\right)\right)-\rho\left(f^{\prime}(0) \zeta^{r}\left(\bar{\mu}_{r}\right)\right)_{i} \leq \rho \bar{\mu}_{r}\left[h_{i}^{\prime}(0)-h_{i}^{\prime}\left(\rho \zeta_{i}^{r}\left(\bar{\mu}_{r}\right)\right)\right] \zeta_{i}^{r}\left(\bar{\mu}_{r}\right), \quad i=1,2,
$$

and,

$$
f_{i}\left(\rho \zeta^{l}\left(\bar{\mu}_{l}\right)\right)-\rho\left(f^{\prime}(0) \zeta^{l}\left(\bar{\mu}_{l}\right)\right)_{i} \leq \rho \bar{\mu}_{l}\left[h_{i}^{\prime}\left(\rho \zeta_{i}^{l}\left(\bar{\mu}_{l}\right)\right)-h_{i}^{\prime}(0)\right] \zeta_{i}^{l}\left(\bar{\mu}_{l}\right), \quad i=1,2
$$

Proposition 3.12. Suppose that the functions $f$ and $h$ are such that $\bar{\mu}_{r}=\bar{\mu}_{l}, \zeta^{r}\left(\bar{\mu}_{r}\right)=\zeta^{l}\left(\bar{\mu}_{l}\right)$, and $\zeta^{r}\left(\bar{\mu}_{r}\right), \zeta^{l}\left(\bar{\mu}_{l}\right)$ are strictly positive. Then a necessary condition for both the right combined condition (3.13) and the left combined condition (3.14) to be satisfied is that the function $f$ satisfies

$$
f_{i}\left(\rho \zeta^{r}\left(\bar{\mu}_{r}\right)\right) \leq \rho\left(f^{\prime}(0) \zeta^{r}\left(\bar{\mu}_{r}\right)\right)_{i} \quad \text { for all } \rho>0, \quad i=1,2
$$


Proof. Introduce the notation

$$
\Lambda_{i}(\rho):=\gamma_{i}(\rho)=\rho\left(f^{\prime}(0) \zeta^{r}\left(\bar{\mu}_{r}\right)\right)_{i}-f_{i}\left(\rho \zeta^{r}\left(\bar{\mu}_{r}\right)\right), \quad \rho>0, \quad i=1,2,
$$

and note that both (3.13) and (3.14) are satisfied if and only if

$$
-\Lambda_{i}(\rho) \leq \rho \bar{\mu}_{r}\left[h_{i}^{\prime}(0)-h_{i}^{\prime}\left(\rho \zeta^{r}\left(\bar{\mu}_{r}\right)\right)\right] \zeta_{i}^{r}\left(\bar{\mu}_{r}\right) \leq \Lambda_{i}(\rho) \text {, for all } \rho>0, i=1,2 .
$$

The result is then immediate from the fact that (3.17) can only hold if $\Lambda_{i}(\rho) \geq 0$ for all $\rho>0$, $i=1,2$, which is equivalent to (3.15).

Remark 3.13. Lemma 3.5 shows that $\bar{\mu}_{r}=\bar{\mu}_{l}$ if the function $f$ is as in the second case in Remark 3.4 (ii), and $\zeta^{r}\left(\bar{\mu}_{r}\right)=\zeta^{l}\left(\bar{\mu}_{l}\right)$ if we also have that $h_{1}^{\prime}(0)=h_{2}^{\prime}(0)$, and Lemma 3.10 gives conditions that ensure $\zeta^{r}\left(\bar{\mu}_{r}\right), \zeta^{l}\left(\bar{\mu}_{l}\right)$ are strictly positive. Note also that the scalar analogue of (3.15) is clearly equivalent to the classical condition (1.3) and an analogue of Proposition 3.12 holds in the scalar case.

The following example illustrates Proposition 3.12.

Example 3.14. Again choose $f: \mathbb{R} \rightarrow \mathbb{R}$ such that $f(u)=u(1-u)(u+\delta)$, where $\delta>0$ (see also Example 2.6 above). If $\delta \geq 1$, this function $f$ satisfies properties $e_{1}-e_{3}$ and (1.3). Then with $\bar{\mu}_{r}=\sqrt{\delta}, \zeta^{r}\left(\bar{\mu}_{r}\right)=1$, (3.17) becomes

$$
-\Lambda(\rho) \leq \rho \sqrt{\delta}\left[h^{\prime}(0)-h^{\prime}(\rho)\right] \leq \Lambda(\rho), \rho>0,
$$

where $\Lambda(\rho)=\rho f^{\prime}(0)-f(\rho)=\rho^{3}+(\delta-1) \rho^{2}>0$. An example of a function $h$ satisfying (3.18) can be constructed by, for instance, taking $\rho \sqrt{\delta}\left[h^{\prime}(0)-h^{\prime}(\rho)\right] \equiv \Lambda(\rho)$, in which case $h^{\prime}(\rho)=$ $A+\frac{\rho(1-\rho-\delta)}{\sqrt{\delta}}$, where $A:=h^{\prime}(0)$, and hence a function $h$ that satisfies (3.18) is

$$
h(\rho)=\frac{(1-\delta) \rho^{2}}{2 \sqrt{\delta}}-\frac{\rho^{3}}{3 \sqrt{\delta}}+A \rho+B, \quad A, B \in \mathbb{R} .
$$

On the other hand, if $0<\delta<1$, the function $f$ does not satisfy condition (1.3), and thus by Proposition 3.12, it is impossible to find a function $h$ which satisfy both the right and left combined conditions (3.13) and (3.14).

Our final example for this section employs a function $f: \mathbb{R}^{2} \rightarrow \mathbb{R}^{2}$, also used in [21, Example 4.1], that falls into the second category in Remark 3.4 (ii) and so has two blocks in the Frobenius form of $f^{\prime}(0)$. This reaction function $f$ is obtained from a competition nonlinearity using a well-known change of variables that converts competition systems to cooperative systems; see [21]. Here we derive conditions on the diffusion coefficient $d_{2}$ and $h_{1}^{\prime}(0), h_{2}^{\prime}(0)$ which are sufficient to allow the construction of a function $h=\left(h_{1}\left(u_{1}\right), h_{2}\left(u_{2}\right)\right)$ such that $h^{\prime}(0)=\operatorname{diag}\left(h_{1}^{\prime}(0), h_{2}^{\prime}(0)\right)$ and both (3.13) and (3.14) will be satisfied. Two separate cases are treated: first, when $h_{1}^{\prime}(0)=h_{2}^{\prime}(0)$, in which case the eigenvectors $\zeta^{r}\left(\bar{\mu}_{r}\right), \zeta^{l}\left(\bar{\mu}_{l}\right)$ are equal, and second, when $h_{1}^{\prime}(0) \neq h_{2}^{\prime}(0)$, in which case $\zeta^{r}\left(\bar{\mu}_{r}\right) \neq \zeta^{l}\left(\bar{\mu}_{l}\right)$. 
Example 3.15. Let $d_{1}=1$ and choose

$$
f\left(u_{1}, u_{2}\right)=\left(\begin{array}{c}
3 u_{1}-4 u_{1}^{2}+u_{1} u_{2} \\
5 u_{2}^{2}-u_{2}+8 u_{1}-4 u_{2}^{3}-8 u_{1} u_{2}
\end{array}\right)
$$

so that

$$
f^{\prime}\left(u_{1}, u_{2}\right)=\left(\begin{array}{cc}
3-8 u_{1}+u_{2} & u_{1} \\
8-8 u_{2} & 10 u_{2}-1-12 u_{2}^{2}-8 u_{1}
\end{array}\right), \quad f^{\prime}(0)=\left(\begin{array}{cc}
3 & 0 \\
8 & -1
\end{array}\right),
$$

and denote $h^{\prime}(0)=\operatorname{diag}(a, b)$, where $a, b \in \mathbb{R}$ with possibly $a \neq b$. There are four solutions of $f\left(u_{1}, u_{2}\right)=0$ with $u_{1}, u_{2} \geq 0$, namely the four equilibria $(0,0),\left(0, \frac{1}{4}\right),(0,1)$ and $(1,1)$. Taking $\beta=$ $(1,1)$, hypotheses $s_{1}-s_{6}$ are clearly satisfied with the minor modification that hypothesis $s_{1}$ holds for all $(u, v) \in[(0,0),(1,1)]$ rather than for all $\left(u_{1}, u_{2}\right)$, which is easily seen to be sufficient for the above theory to apply because all solutions $\left(u_{1}, u_{2}\right)$ of (3.1) considered here lie between the equilibria $(0,0)$ and $\beta=(1,1)$.

Then $\bar{\mu}_{r}=\bar{\mu}_{l}=\sqrt{3}$, and eigenvectors $\zeta^{r}\left(\bar{\mu}_{r}\right), \zeta^{l}\left(\bar{\mu}_{l}\right)$ for $\gamma_{1}\left(\bar{\mu}_{r}\right)=6+\sqrt{3} a, \gamma_{1}\left(\bar{\mu}_{l}\right)=6-\sqrt{3} a$ are

$$
\zeta^{r}\left(\bar{\mu}_{r}\right)=\left(\begin{array}{c}
1 \\
8 /\left(7+\sqrt{3}(a-b)-3 d_{2}\right)
\end{array}\right)=\left(\begin{array}{c}
1 \\
\alpha_{2}
\end{array}\right), \zeta^{l}\left(\bar{\mu}_{l}\right)=\left(\begin{array}{c}
1 \\
8 /\left(7-\sqrt{3}(a-b)-3 d_{2}\right)
\end{array}\right)=\left(\begin{array}{c}
1 \\
\hat{\alpha}_{2}
\end{array}\right) .
$$

Provided $d_{2}<7 / 3$, it follows from Lemma 3.10 (and by inspection) that if $a, b$ satisfy

$$
\frac{-\left(7-3 d_{2}\right)}{\sqrt{3}}<a-b<\frac{\left(7-3 d_{2}\right)}{\sqrt{3}}
$$

then the eigenvectors $\zeta^{r}\left(\bar{\mu}_{r}\right), \zeta^{l}\left(\bar{\mu}_{l}\right)$ are strictly positive and (3.8), (3.10) are satisfied.

Now define $\eta:=a-b$ and consider the cases $\eta=0$ and $\eta \neq 0$. Suppose $\eta=0$. Then the eigenvectors $\zeta^{r}\left(\bar{\mu}_{r}\right), \zeta^{l}\left(\bar{\mu}_{l}\right)$ are equal (cf. Lemma 3.5). Moreover, provided $d_{2}$ satisfies the stricter restriction that $d_{2} \leq 2 / 3$, the function $f$ satisfies the necessary condition (3.15) of Proposition 3.12, in which case it is clearly possible to construct functions $h_{1}, h_{2}$ for which both (3.13), (3.14) hold by using a similar method to that in our explicit construction of $h$ in Example 3.14.

For $\eta \neq 0$, to have that both conditions (3.13), (3.14) are satisfied for a given $h_{1}$, we require

$$
h_{1}^{\prime}(\rho) \leq a-\left(\frac{\rho\left(-4+\alpha_{2}\right)}{\sqrt{3}}\right), \quad h_{1}^{\prime}(\rho) \geq a+\left(\frac{\rho\left(-4+\hat{\alpha}_{2}\right)}{\sqrt{3}}\right), \quad \rho>0,
$$

thus we need

$$
\frac{t}{\sqrt{3}}\left(\hat{\alpha}_{2}-4\right) \leq h_{1}^{\prime}(t)-a \leq \frac{t}{\sqrt{3}}\left(-\alpha_{2}+4\right), \quad t>0,
$$

which can be satisfied if $\hat{\alpha}_{2}-4 \leq-\alpha_{2}+4$, that is equivalent to requiring

$$
9 d_{2}^{2}-36 d_{2}+35-3 \eta^{2} \geq 0
$$


Since (3.19) holds if $d_{2} \leq\left(6-\sqrt{1+3 \eta^{2}}\right) / 3$, a function $h_{1}$ satisfying the first inequality in each of (3.13), (3.14) can be constructed for such $d_{2}, \eta$. Note that the larger values of $d_{2}$ that satisfy (3.19) violate the additional requirement that $d_{2}<7 / 3$, and that it is clearly necessary to have $\eta^{2}<35 / 3$ to be able to construct $h_{1}$ for some $d_{2}>0$.

Then for the existence of a function $h_{2}$ such that both (3.13), (3.14) are satisfied, we need

$$
h_{2}^{\prime}\left(\rho \alpha_{2}\right) \leq b-\left(\frac{5\left(\rho \alpha_{2}\right)-4\left(\rho \alpha_{2}\right)^{2}-8 \rho}{\sqrt{3}}\right), \quad h_{2}^{\prime}\left(\rho \hat{\alpha}_{2}\right) \geq b+\left(\frac{5\left(\rho \hat{\alpha}_{2}\right)-4\left(\rho \hat{\alpha}_{2}\right)^{2}-8 \rho}{\sqrt{3}}\right), \quad \rho>0 .
$$

Thus we require that

$$
\frac{5 t-4 t^{2}-8 t / \hat{\alpha}_{2}}{\sqrt{3}} \leq h_{2}^{\prime}(t)-b \leq-\left(\frac{5 t-4 t^{2}-8 t / \alpha_{2}}{\sqrt{3}}\right), \quad t>0,
$$

which can hold if $-2 t\left(2+4 t-3 d_{2}\right) \leq 0$ for all $t>0$, and if $d_{2} \leq(2+4 t) / 3$ for all $t>0$. Hence a function $h_{2}$ satisfying (3.13), (3.14) can always be constructed, regardless of the value of $\eta$, provided $d_{2} \leq 2 / 3$.

Thus we have shown that for this choice of $f: \mathbb{R}^{2} \rightarrow \mathbb{R}^{2}$, if $0 \leq|a-b| \leq \sqrt{5}$, a sufficient condition to be able to find a function $h=\left(h_{1}, h_{2}\right)$ so that both combined conditions (3.13), (3.14) are satisfied and $h^{\prime}(0)=\operatorname{diag}(a, b)$ is $d_{2} \leq 2 / 3$, whereas if $\sqrt{5} \leq|a-b|<\sqrt{35 / 3}$, a function $h=\left(h_{1}, h_{2}\right)$ for which (3.13), (3.14) both hold and $h^{\prime}(0)=\operatorname{diag}(a, b)$ can be constructed provided

$$
d_{2} \leq \frac{6-\sqrt{1+3(a-b)^{2}}}{3} .
$$

We conclude by noting that Example 3.15 illustrates a possible interplay between the diffusion coefficient $d_{2}$ and the linearisation of the convective term $h^{\prime}(0)=\operatorname{diag}\left(h_{1}^{\prime}(0), h_{2}^{\prime}(0)\right)$ in determining whether a system can be right and/or left linearly determinate. It would be interesting to explore a more general theory of such interplay between the rôles diffusion and convection.

\section{Acknowledgement}

We are very grateful to the referee for his/her careful reading of the manuscript and valuable comments, and in particular, for suggesting the idea on which Example 2.7 is based. A. Al-Kiffai thanks the Iraqi Ministry of Higher Education and Scientific Research (MOHESR) for financial support in the form of a PhD studentship.

\section{References}

[1] A. Al-Kiffai, The role of convection on spreading speeds and linear determinacy for reaction-diffusion-convection systems, PhD Thesis, Swansea University, submitted. 
[2] R. D. Benguria, M. C. Depassier and V. Mendez, Speed of travelling waves in reaction-diffusion equations, Phys. Rev. E, 3 (2001), 109-110.

[3] R. D. Benguria, M. C. Depassier and V. Mendez, Minimal speed of fronts of reaction-convection-diffusion equations, Phys. Rev. E, 69 (2004), 031106, p 7.

[4] C. Castillo-Chavez, B. Li, and H. Wang, Some recent developments on linear determinacy, Math. Biosci. Eng., 10 (2013), 1419-1436.

[5] E. C. M. Crooks, Travelling fronts for monostable reaction-diffusion systems with gradient-dependence, Advances in Differential Equations, 8 (2003), No.3, 279-314.

[6] R. A. Fisher, The wave of advance of advantageous genes, Ann. Eugenics, 7 (1937), 355-369.

[7] B. H. Gilding and R. Kersner, Travelling waves in nonlinear diffusion-convection-reaction, Progress in Nonlinear Differential Equations and their Applications, 60, Birkhäuser Verlag, Basel, (2004).

[8] K. P. Hadeler and F. Rothe, Travelling fronts in nonlinear diffusion equations, J. Math. Biol., 2 (1975), $251-263$.

[9] E. F. Keller and L. A. Segel, Model for chemotaxis, J. Theor. Biol., 30 (1971), 225-234.

[10] A. N. Kolmogorov, I. Petrowskii, N. Piscounov, Etude de l'equation de la diffusion avec croissance de la quantité de matière et son application à un problème biologique, Moscow Univ. Math. Bull., 1 (1937), 1-25.

[11] B. Li, H. F. Weinberger, M. A. Lewis, Spreading speeds as slowest wave speeds for cooperative systems, Mathematical Biosciences, 196 (2005), 82-98.

[12] X. Liang and X. Zhao, Asymptotic speed of spread and traveling waves for monotone semiflows with applications, Commun. Pure Appl. Math., 60 (2007), 1-40.

[13] M. Lucia, C. Muratov and M. Novaga, Linear vs. nonlinear selection for the propagation speed of the solutions of scalar reaction-diffusion equations invading an unstable equilibrium, Comm. Pure Appl. Math., 57 (2004), 616-636.

[14] R. Lui, Biological growth and spread modeled by systems of recursions, I Mathematical theory, Mathematical Bioscience, 93 (1989), 269-295.

[15] L. Malaguti and C. Marcelli, Travelling wave fronts in reaction-diffusion equation with convection effects and non-regular terms, Math., Nach., 242 (2002), 148-164.

[16] J. D. Murray, Mathematical biology, 2nd, corrected edition, Biomathematics Texts, 19 (1989).

[17] A. H. Roger and R. J. Charles, Matrix Analysis, Cambridge University Press, 2nd Edition, 2013.

[18] E. Seneta Non-negative Matrices, an Introduction to Theory and Applications, George Allen and Unwin Ltd, London, 1973.

[19] A. I. Vol'pert and V. A. Vol'pert, Traveling-wave Solutions of Parabolic Systems, vol:140 of Translations of Mathematical Monographs, American Mathematical Society, Providence, R.I., 1994.

[20] H. Weinberger, On sufficient condition for a linearly determinate spreading speed, Discrete Cont. Dyn. Syst. B, 17 (2012), 2267-2280.

[21] H. F. Weinberger, M. A. Lewis, B. Li, Analysis of linear determinacy for spread in cooperative models, J. Math. Biol., 45 (2002), 183-218.

Department of Mathematics, College of Science, Swansea University, Swansea SA2 8PP, U.K.

College of Education for Girls, Kufa University, Al-Najaf Al-Ashraf, Iraq.

E-mail: 717278@swansea.ac.uk

Department of Mathematics, College of Science, Swansea University, Swansea SA2 8PP, U.K.

E-mail: e.c.m.crooks@swansea.ac.uk 